\title{
LncRNA MEG3 rs3087918 was associated with a decreased breast cancer risk in a Chinese population: a case-control study
}

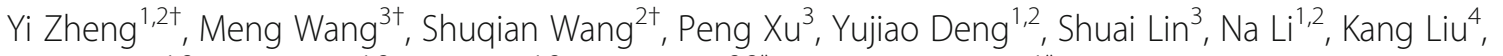
Yuyao Zhu ${ }^{1,2}$, Zhen Zhai ${ }^{1,2}$, Ying Wu ${ }^{1,2}$, Zhijun Dai ${ }^{2,3^{*}}$ and Gaixia Zhu ${ }^{1 *}$

\begin{abstract}
Background: LncRNA MEG3 expressed abnormally in various cancers including breast cancer, but no studies reported the correlation between MEG3 SNPs and breast cancer susceptibility among Chinese women.

Methods: This study is aimed to explore the association between three SNPs of MEG3 (rs3087918, rs7158663, rs11160608) and breast cancer. The study is a population-based case-control study including 434 breast cancer patients and 700 healthy controls. Genotyping was performed using Sequenom MassArray technique. Function prediction of rs3087918 were based on RNAfold and IncRNASNP2 databases.

Results: Pooled analysis indicated that rs3087918 was related to a decreased risk of breast cancer [GG vs. TT: OR $(95 \%)=0.67(0.45-0.99), P=0.042 ; G G$ vs. TT + TG: OR (95\%) $=0.69(0.48-0.99), P=0.046]$, especially for women aged $<=49$ [GG vs. T: OR (95\%) =0.40(0.22-0.73), $P=0.02$ ]. Comparison between case groups showed genotype GG and TG/GG of rs3087918 were associated with her-2 receptor expression [GG vs. TT: OR (95\%) $=2.37(1.24-4.63), P=0.010$; TG + GG vs. TT: OR (95\%) = 1.50(1.01-2.24), $P=0.045]$. We didn't find statistical significance for rs $11160608, \mathrm{rs} 7158663$ and breast cancer. Structure prediction based on RNAfold found rs3087918 may influence the secondary structure of MEG3. The results based on IncRNASNP2 indicated that rs3087918 may gain the targets of hsa-miR-1203 to MEG3, while loss the target of hsa-miR-139-3p and hsa-miR-5091 to MEG3.
\end{abstract}

Conclusions: MEG3 rs3087918 was associated with a decreased risk of breast cancer. MEG3 haplotype TCG may increase the risk of breast cancer.

Keywords: MEG3, SNP, Breast cancer, Case-control study, miRNA

\section{Background}

Breast cancer (BC) is a serious threat to women's health. According to American cancer statistics 2020 [1], there will be an estimated 276,480 new BC cases and 42,170 $\mathrm{BC}$ related death in 2020. For females, BC is the most

\footnotetext{
*Correspondence: dzj0911@126.com; 306425776@qq.com

${ }^{\dagger}{ }^{\top} \mathrm{i}$ Zheng, Meng Wang and Shuqian Wang contributed equally to this work. ${ }^{2}$ Department of Breast Surgery, The First Affiliated Hospital, College of Medicine, Zhejiang University, Hangzhou 310003, China

'Department of Obstetrics and Gynecology, The Second Affiliated Hospital of Xi'an Jiaotong University, Xi'an 710004, China

Full list of author information is available at the end of the article
}

common diagnosed cancer ( $24.2 \%$ of the total cases) and the leading cause of cancer death $(15.0 \%$ of the total cancer death). Although epidemiological studies have identified several risk factors involved in $\mathrm{BC}$, such as age, hormonal state, and family history [2], the pathogenesis of $\mathrm{BC}$ is still unclear. $\mathrm{BC}$ is a complex and genetically heterogeneous disease in which genetic changes such as abnormal amplification of oncogenes, or deletion/mutation of tumor suppressor genes, play a substantial role [3-5].

C C The Author(s). 2020 Open Access This article is licensed under a Creative Commons Attribution 4.0 International License, which permits use, sharing, adaptation, distribution and reproduction in any medium or format, as long as you give appropriate credit to the original author(s) and the source, provide a link to the Creative Commons licence, and indicate if changes were made. The images or other third party material in this article are included in the article's Creative Commons licence, unless indicated otherwise in a credit line to the material. If material is not included in the article's Creative Commons licence and your intended use is not permitted by statutory regulation or exceeds the permitted use, you will need to obtain permission directly from the copyright holder. To view a copy of this licence, visit http://creativecommons.org/licenses/by/4.0/. The Creative Commons Public Domain Dedication waiver (http://creativecommons.org/publicdomain/zero/1.0/) applies to the data made available in this article, unless otherwise stated in a credit line to the data. 
Maternally expressed gene 3 (MEG3) is an imprinted gene located at chromosome 14q32.3 in humans, encoding a long non-coding RNA (lncRNA) belonging to the imprinted DLK1-MEG3 regions [6]. This region contains at least three paternally expressed protein coding genes and numerous maternally expressed noncoding RNAs [7]. The imprinted expression of these genes was related to cell development and growth [8], and experiments in vitro indicated MEG3 can suppress the proliferation of human cancer cells lines [9]. Researchers found loss of MEG3 related to a variety of human cancers, such as gastric [10], cervical [11], and breast [12] cancer. MEG3 can inhibit the occurrence of tumor through various aspects. Firstly, MEG3 can inhibit the proliferation of tumor cells and consequently induce apoptosis, which has been confirmed by in vitro experiments and animal models [13]. Secondly, MEG3 plays a role in epigenetic regulation and can alter the function of cancer cells by affecting DNA methylation and regulating the functions of snoRNA and miRNA [14, 15]. Moreover, MEG3 is involved in the regulation of many tumor-related signaling pathways, including p53, MDM2, and pRb pathway [16].

Single-nucleotide polymorphism (SNP) mainly refers to the DNA sequence polymorphism caused by the variation of a single nucleotide at the genome level. It is the most common genetic variant in the human genome, accounting for $90 \%$ of all known polymorphisms [17]. To date, Genome Wide Association Study (GWAS) and multiple large-scale sequencing have identified many SNPs in more than 70 genes associated with breast cancer $[18,19]$. SNP has been considered a potential biomarker of genetic background to predict risk, progression, and treatment response to various diseases. Previous investigation indicated that several SNPs in MEG3 genes are associated with breast cancer susceptibility [20]. However, there are no investigation to explore the relationship between MEG3 polymorphisms and breast cancer among Chinese women. In this study, we genotyped three polymorphisms (rs3087918, rs11160608 rs7158663) in MEG3 gene based on $434 \mathrm{BC}$ patients and 700 healthy controls, to explore their relationship with breast cancer.

\section{Methods}

\section{Study subjects}

In total, 1134 females were recruited for this population-based case-control study. Among these, 434 breast cancers were enrolled in the Department of Oncology, the Second Affiliated Hospital, Xi'an Jiaotong University, from 2013 to 2015. Seven hundred healthy females were randomly recruited from medical center of the same hospital during the same period. All BC patients were diagnosed by pathology and detailed immunohistochemical analysis. $\mathrm{BC}$ patients who had a history of other malignant diseases or receiving chemotherapy or radiotherapy were excluded. The controls were matched to cases by age $( \pm$ 2 years) and had no history of malignant tumors, no history of chemoradiotherapy, no obvious abnormality in blood routine examination. The protocol of this study was approved by the Ethics Committee of the Second Affiliated Hospital of Xi'an Jiaotong University Shaanxi Province (Xi'an, China). All patients gave written informed consent prior to participation in the study.

\section{SNP selection and genotyping}

SNPs were selected from NCBI dbSNP database (https://www.ncbi.nlm.nih.gov/projects/SNP) and relevant literature [20-22] according to the following criteria. First, the minor allele frequency (MAF) was no less than 0.05 among Chinese population. Secondly, the SNPs located in the $5^{\prime}$ - flanking region, $5^{\prime}$ untranslated region, $3^{\prime}$ untranslated region, and exon of MEG3 gene. We finally chose three MEG3 SNPs rs3087918, rs11160608 rs7158663 to study. Peripheral blood samples were collected in EDTA-coated tubes and conserved at $-80^{\circ} \mathrm{C}$. Genome DNA were extracted from whole blood samples using ComWin BloodGen Mini Kit (QIAGEN, China, Beijing). Ultraviolet spectrophotometer (Nanodrop, Thermo Scientific, Waltham, MA) was utilized to measure the purity and concentration of extracted DNA. We designed multiplexed SNP MassEXTEND assay using Sequenom MassARRAY Assay Design 3.0 software. DNA samples were genotyped by Sequenom MassARRAY RS1000 according to the standard protocol. The primers applied for the three SNPs were shown in Supplemental Table S1.

\section{Statistical analysis}

The HWE of the three SNPs were calculated using Fisher's exact test in controls group. Student's $t$ test was adopted to evaluated the difference of age distribution and body mass index (BMI) between BC patients and healthy controls. Two-sided Pearson's chi-square tests were applied to access the differences in the categorical variables between cases and controls, such as age $(<=49$ and $>49$ ), BMI, menstrual-status, and allelic frequencies. $P<0.05$ was considered statistically significant. We also calculated odds ratios (ORs) and 95\% confidence intervals (CIs) using logistic regression analysis. Haplotype analysis were conducted by Haploview 4.2. Other statistical analyses were performed using the version $\mathrm{R} 3.5 .2$ software. 


\section{Function prediction based on databases}

We used RNAfold (http://rna.tbi.univie.ac.at//cgi-bin/ RNAWebSuite/RNAfold.cgi) and LncRNASNP2 (http:// bioinfo.life.hust.edu.cn/lncRNASNP/) database to predict the effect of SNP on MEG3. RNAfold is a classic database to predict RNAs structure. Free energy represents the amount of energy that needs to be injected to change the structure. The smaller the corresponding value is, the more stable the structure will be. LncRNASNP2 is a novel database containing 7,260,238 SNPs on 141,353 human lncRNA transcripts and 3,921, 448 SNPs on 117,405 mouse lncRNA transcripts [23]. We used this database to predict the potential function of the MEG3 polymorphisms.

\section{Results}

\section{Demographical and clinical information of study population}

This study contained $434 \mathrm{BC}$ cases and 700 healthy control. All the subjects were Han Chinses women from northwest China. There were no statistically significant differences in age distribution, BMI and menopausal status between the patients and the control group. The detail demographical and clinical information was display in Table 1. BMI was a statistical index to estimate the

Table 1 Demographic information

\begin{tabular}{|c|c|c|c|}
\hline Characteristics & Cases (\%) & Controls (\%) & $P$ value \\
\hline Number & 434 & 700 & \\
\hline Age $($ mean $\pm S D)$ & $51.95 \pm 10.35$ & $51.83 \pm 17.28$ & $0.879^{a}$ \\
\hline$\leqq 49$ & $180(41.5)$ & $298(42.6)$ & \\
\hline$>49$ & $254(58.5)$ & $402(57.4)$ & 0.716 \\
\hline $\mathrm{BMl}, \mathrm{kg} / \mathrm{m} 2($ mean $\pm \mathrm{SD})$ & $22.38 \pm 2.61$ & $22.71 \pm 4.00$ & $0.084^{\mathrm{a}}$ \\
\hline \multicolumn{4}{|l|}{ Menopausal status } \\
\hline Premenopausal & $157(36.2)$ & $188(41.8)$ & \\
\hline Postmenopausal & $277(63.8)$ & $262(58.2)$ & 0.506 \\
\hline \multicolumn{4}{|l|}{ TNM Stage } \\
\hline । & $114(26.3)$ & - & - \\
\hline$\|$ & $192(44.2)$ & & - \\
\hline III & $89(20.5)$ & - & - \\
\hline IV & $39(9)$ & - & - \\
\hline \multicolumn{4}{|c|}{ Immunohistochemistry results } \\
\hline \multirow[t]{2}{*}{ ER } & $142(32.7)$ & - & - \\
\hline & $292(67.3)$ & - & - \\
\hline \multirow[t]{2}{*}{$P R$} & $189(43.5)$ & - & - \\
\hline & $245(56.5)$ & - & - \\
\hline \multirow[t]{2}{*}{ Her-2 } & $250(57.6)$ & - & - \\
\hline & $184(42.4)$ & - & - \\
\hline
\end{tabular}

BMI: body mass index, ER: estrogen receptor, $P R$ : progesterone receptor, Her-2: human epidermal growth factor receptor-2

a Student's t-test body fat in people of any age. In this study, BMI was divided into four levels (underweight, normal weight, overweight, and obese) based on Chinese reference standard.

\section{The associations between MEG3 SNPs and BC risk}

Three SNP in MEG3 gene (rs3087918, rs11160608 rs7158663) were genotyped in all recruited subjects, and their detected rate were 99.1, 99.2 and 99.4\%, respectively. The genotype distribution of the three polymorphisms in control groups accorded with HWE (rs11160608: $\quad P_{H W E}=0.844 ; \quad$ rs3087918: $P_{H W E}=0.968$; rs7158663: $\left.P_{H W E}=0.334\right)$. We didn't find statistical significance for rs11160608, rs7158663 and breast cancer ( $P>0.05$ in all genetic models). Pooled analysis indicated that rs3087918 was related to a decreased risk of breast cancer [GG vs. TT: OR $(95 \% \mathrm{CI})=0.67(0.45-0.99), P=$ 0.042; GG vs. TT + TG: OR $(95 \% \mathrm{CI})=0.69(0.48-0.99)$, $P=0.046]$. The detail results were showed in Table 2 .

\section{Stratified analysis by age, BMI and menopausal status}

Then, we conducted stratified analysis based on age, BMI and menopausal status to further explore their effect on relationship between $\mathrm{BC}$ susceptibility and the three SNPs in MEG3. BMI was divided into two levels $\left(\mathrm{BMI}<24 \mathrm{~kg} / \mathrm{m}^{2}\right.$ and $\left.\mathrm{BMI}>=24 \mathrm{~kg} / \mathrm{m}^{2}\right)$. No association was found between rs11160608, rs7158663 and breast cancer when stratified by age, BMI and menopausal status (Supplemental Table S2). Rs3087918 was related to a reduced susceptibility for women aged $<=49 \quad[\mathrm{GG}$ vs. TT: OR $(95 \% \mathrm{CI})=$ 0.40(0.22-0.73), $P=0.02$ ] (Table 3).

\section{Relationship between MEG3 rs3087918 and clinical characteristics of BC}

To further explore the effect of rs3087918 loci and clinicopathological information on BC susceptibility, correlation analysis was conducted in the cases group defined by age, BMI, menopausal status, tumor size, metastasis, clinical stage, ER/PR status and Her-2. As showed in Table 4, there is a significant association of the GG genotype with tumor size according to the $95 \%$ CI $(1.01-3.92)$, while the $P$ value of tumor size is 0.05 . In this study, $P<0.05$ was considered statistically significant. Thus, we considered there was no association found between GG genotype of rs3087918 and tumor size. This is a controversial result that needs further study to clarify. GG and TG + GG genotypes were associated with the over-expression of Her-2 [GG vs. TT: OR $(95 \% \mathrm{CI})=2.37(1.24-4.63), P=0.010$; TG + GG vs. TT: OR $(95 \% \mathrm{CI})=1.50(1.01-2.24), P=$ 0.045]. We further divided the cases into luminal, Her-2 and triple negative breast cancer (TNBC) groups according to molecular classification. However, 
Table 2 Association between MEG3 gene polymorphisms and risk of breast cancer (rs11160608, rs3087918, rs7158663)

\begin{tabular}{|c|c|c|c|c|c|}
\hline $\begin{array}{l}\text { SNPs genetic } \\
\text { model }\end{array}$ & Genotype & $\begin{array}{l}\text { Cases } \\
(\%) \\
N=434\end{array}$ & $\begin{array}{l}\text { Controls } \\
(\%) \\
N=700\end{array}$ & $\begin{array}{l}\text { OR } \\
(95 \% \mathrm{Cl})\end{array}$ & $P$ value \\
\hline \multicolumn{6}{|l|}{ rs11160608 } \\
\hline \multirow[t]{3}{*}{ Co-dominant } & $\mathrm{AA}$ & $\begin{array}{l}126 \\
(29.7)\end{array}$ & $227(32.4)$ & reference & \\
\hline & $A C$ & $\begin{array}{l}218 \\
(51.4)\end{array}$ & $341(48.7)$ & $\begin{array}{l}1.15 \\
(0.87-1.52)\end{array}$ & 0.316 \\
\hline & $\mathrm{CC}$ & $\begin{array}{l}80 \\
(18.9)\end{array}$ & $132(18.9)$ & $\begin{array}{l}1.09 \\
(0.77-1.55)\end{array}$ & 0.625 \\
\hline \multirow[t]{2}{*}{ Dominant } & $\mathrm{AA}$ & $\begin{array}{l}126 \\
(29.7)\end{array}$ & $227(32.4)$ & reference & \\
\hline & $A C+C C$ & $\begin{array}{l}298 \\
(70.3)\end{array}$ & $473(67.6)$ & $\begin{array}{l}1.14 \\
(0.87-1.48)\end{array}$ & 0.342 \\
\hline \multirow[t]{2}{*}{ Recessive } & $A A+A C$ & $\begin{array}{l}344 \\
(81.1)\end{array}$ & $568(81.1)$ & reference & \\
\hline & $\mathrm{CC}$ & $\begin{array}{l}80 \\
(18.9)\end{array}$ & $132(18.9)$ & $\begin{array}{l}1.00 \\
(0.74-1.36)\end{array}$ & 0.996 \\
\hline \multirow[t]{2}{*}{ Allele } & A & $\begin{array}{l}470 \\
(55.4)\end{array}$ & 795 (56.8) & reference & \\
\hline & C & $\begin{array}{l}378 \\
(44.6)\end{array}$ & $605(43.2)$ & $\begin{array}{l}1.06 \\
(0.89-1.26)\end{array}$ & 0.528 \\
\hline
\end{tabular}

rs3087918

\begin{tabular}{|c|c|c|c|c|c|}
\hline \multirow[t]{3}{*}{ Co-dominant } & $\pi$ & $\begin{array}{l}171 \\
(40.2)\end{array}$ & $259(37.0)$ & reference & \\
\hline & $\mathrm{TG}$ & $\begin{array}{l}207 \\
(48.7)\end{array}$ & $334(47.7)$ & $\begin{array}{l}0.94 \\
(0.72-1.22)\end{array}$ & 0.633 \\
\hline & GG & $\begin{array}{l}47 \\
(11.1)\end{array}$ & $107(15.3)$ & $\begin{array}{l}0.67 \\
(0.45-0.99)\end{array}$ & $0.042^{*}$ \\
\hline \multirow[t]{2}{*}{ Dominate } & $\pi$ & $\begin{array}{l}171 \\
(40.2)\end{array}$ & $259(37.0)$ & reference & \\
\hline & $\mathrm{TG}+\mathrm{GG}$ & $\begin{array}{l}254 \\
(59.8)\end{array}$ & $441(63.0)$ & $\begin{array}{l}0.87 \\
(0.68-1.12)\end{array}$ & 0.279 \\
\hline \multirow[t]{2}{*}{ Recessive } & $\Pi+\mathrm{TG}$ & $\begin{array}{l}378 \\
(88.9)\end{array}$ & $593(84.7)$ & reference & \\
\hline & GG & $\begin{array}{l}47 \\
(11.1)\end{array}$ & $107(15.3)$ & $\begin{array}{l}0.69 \\
(0.48-0.99)\end{array}$ & $0.046^{*}$ \\
\hline \multirow[t]{2}{*}{ Allele } & $\mathrm{T}$ & $\begin{array}{l}549 \\
(64.6)\end{array}$ & $852(60.9)$ & reference & \\
\hline & G & $\begin{array}{l}301 \\
(35.4)\end{array}$ & $548(39.1)$ & $\begin{array}{l}0.85 \\
(0.71-1.02)\end{array}$ & 0.077 \\
\hline \multicolumn{6}{|l|}{ s7158663 } \\
\hline \multirow[t]{3}{*}{ Co-dominate } & GG & $\begin{array}{l}224 \\
(52.5)\end{array}$ & $403(0.6)$ & reference & \\
\hline & GA & $\begin{array}{l}170 \\
(39.8)\end{array}$ & $250(0.4)$ & $\begin{array}{l}1.22 \\
(0.95-1.58)\end{array}$ & 0.12 \\
\hline & AA & $33(7.7)$ & $47(0.1)$ & $\begin{array}{l}1.26 \\
(0.79-2.03)\end{array}$ & 0.333 \\
\hline \multirow[t]{2}{*}{ Dominate } & GG & $\begin{array}{l}224 \\
(52.5)\end{array}$ & $403(0.6)$ & reference & \\
\hline & $\mathrm{GA}+\mathrm{AA}$ & $\begin{array}{l}203 \\
(47.5)\end{array}$ & $297(0.4)$ & $\begin{array}{l}1.23 \\
(0.97-1.57)\end{array}$ & 0.094 \\
\hline Recessive & $\mathrm{GG}+\mathrm{GA}$ & $\begin{array}{l}394 \\
(92.3)\end{array}$ & $653(0.9)$ & reference & \\
\hline
\end{tabular}

Table 2 Association between MEG3 gene polymorphisms and risk of breast cancer (rs11160608, rs3087918, rs7158663)

\begin{tabular}{|c|c|c|c|c|c|}
\hline $\begin{array}{l}\text { SNPs genetic } \\
\text { model }\end{array}$ & Genotype & $\begin{array}{l}\text { Cases } \\
(\%) \\
N=434\end{array}$ & $\begin{array}{l}\text { Controls } \\
(\%) \\
N=700\end{array}$ & $\begin{array}{l}\text { OR } \\
(95 \% \mathrm{Cl})\end{array}$ & $P$ value \\
\hline & $\overline{A A}$ & $33(7.7)$ & $47(0.1)$ & $\begin{array}{l}1.16 \\
(0.73-1.85)\end{array}$ & 0.52 \\
\hline \multirow[t]{2}{*}{ Allele } & G & $\begin{array}{l}618 \\
(72.4)\end{array}$ & $\begin{array}{l}1056 \\
(75.4)\end{array}$ & reference & \\
\hline & A & $\begin{array}{l}236 \\
(27.6)\end{array}$ & $344(24.6)$ & $\begin{array}{l}1.17 \\
(0.97-1.42)\end{array}$ & 0.107 \\
\hline
\end{tabular}

OR: odds ratio, Cl: confidence interval

*The $P$ Value $<0.05$

we found no association between three SNPs of MEG3 and the different molecular typing states of BC (Supplemental Table S3).

\section{Haplotype analysis of MEG3 SNPs and associations with the risk of $\mathrm{BC}$}

To explore the combined effect the three SNPs in MEG3, we performed haplotype analysis by Haploview. The results of the haploid analysis indicated that TCG haplotype may increase the risk of breast cancer compared with the wild haplotype TAG [OR $(95 \% \mathrm{CI})=2.97(1.66-5.31), P<0.001]$. Other haplotypes showed no association with BC (Table 5). The order of the three SNPs was rs3087918, rs11160608 and rs7158663.

\section{The function prediction of the rs3087918 in MEG3}

We used RNAfold (http://rna.tbi.univie.ac.at//cgi-bin/ RNAWebSuite/RNAfold.cgi) and LncRNASNP2 (http:// bioinfo.life.hust.edu.cn/lncRNASNP/) database to predict the potential function of rs3078918. The centroid secondary structure of rs3087918 was shown in Fig. 1, we learned that mutant allele " $G$ " would significantly change the centroid secondary structure of MEG3. Moreover, its minimum free energy was change from - $28.87 \mathrm{kcal}$ to $26.90 \mathrm{kcal} / \mathrm{mol}$, which suggests rs3087918 may increase the structural stability of MEG3. The results of LncRNASNP2 indicated that rs3087918 may gain the targets of hsa-miR-1203 to MEG3 (lncRNA ID: NONHSAT039760.2), while loss the target of hsa-miR-139-3p and hsa-miR-5091 to MEG3 (See Supplemental Table S4 and Figure S1).

\section{Discussion}

The occurrence of breast cancer is a result of a longterm complex interaction between individual genetic background and environmental exposure factors. As the most common type of genetic mutation, SNP is of great significance for breast cancer risk, diagnosis, individualized treatment and prognosis prediction. 
Table 3 Stratified Analysis of rs3087918 by age, BMl and menopausal status

\begin{tabular}{|c|c|c|c|c|}
\hline \multirow[t]{2}{*}{ Group } & \multicolumn{4}{|c|}{ rs3087918 (Case/Control) } \\
\hline & $\pi$ & TG & GG & $\mathrm{TG}+\mathrm{GG}$ \\
\hline \multicolumn{5}{|l|}{ Age } \\
\hline$<=49$ & $69 / 93$ & $87 / 141$ & $19 / 64$ & $106 / 205$ \\
\hline $\mathrm{OR}(95 \% \mathrm{Cl})$ & $\begin{array}{l}1.00 \\
\text { (reference) }\end{array}$ & $\begin{array}{l}0.83(0.55- \\
1.25)\end{array}$ & $\begin{array}{l}0.40(0.22- \\
0.73)\end{array}$ & $\begin{array}{l}0.70(0.47- \\
1.03)\end{array}$ \\
\hline$P$-value & & 0.378 & $0.002^{*}$ & 0.069 \\
\hline$>49$ & $102 / 166$ & $120 / 193$ & $28 / 43$ & $148 / 236$ \\
\hline $\mathrm{OR}(95 \% \mathrm{Cl})$ & $\begin{array}{l}1.00 \\
\text { (reference) }\end{array}$ & $\begin{array}{l}1.01(0.72- \\
1.42)\end{array}$ & $\begin{array}{l}1.06(0.62- \\
1.81)\end{array}$ & $\begin{array}{l}1.02(0.74- \\
1.41)\end{array}$ \\
\hline$P$-value & & 0.945 & 0.832 & 0.901 \\
\hline \multicolumn{5}{|l|}{ BMI (kg/m2) } \\
\hline$<24$ & $134 / 206$ & $147 / 254$ & $35 / 74$ & $182 / 328$ \\
\hline $\mathrm{OR}(95 \% \mathrm{Cl})$ & $\begin{array}{l}1.00 \\
\text { (reference) }\end{array}$ & $\begin{array}{l}0.89(0.66- \\
1.20)\end{array}$ & $\begin{array}{l}0.73(0.46- \\
1.15)\end{array}$ & $\begin{array}{l}0.85(0.64- \\
1.13)\end{array}$ \\
\hline$P$-value & & 0.441 & 0.171 & 0.271 \\
\hline$>=24$ & $37 / 53$ & $60 / 80$ & $12 / 33$ & $72 / 113$ \\
\hline OR(95\%Cl) & $\begin{array}{l}1.00 \\
\text { (reference) }\end{array}$ & $\begin{array}{l}1.07(0.63- \\
1.84)\end{array}$ & $\begin{array}{l}0.52(0.24- \\
1.14)\end{array}$ & $\begin{array}{l}0.91(0.55- \\
1.53)\end{array}$ \\
\hline P-value & & 0.794 & 0.100 & 0.727 \\
\hline \multicolumn{5}{|l|}{ Menstrual-status } \\
\hline postmenopausal & $114 / 167$ & $128 / 201$ & $29 / 65$ & $157 / 266$ \\
\hline OR(95\%Cl) & $\begin{array}{l}1.00 \\
\text { (reference) }\end{array}$ & $\begin{array}{l}0.93(0.67- \\
1.29)\end{array}$ & $\begin{array}{l}0.65(0.40- \\
1.08)\end{array}$ & $\begin{array}{l}0.87(0.64- \\
1.18)\end{array}$ \\
\hline$P$-value & & 0.675 & 0.093 & 0.356 \\
\hline menstruating & $57 / 92$ & 79/133 & $18 / 42$ & $97 / 175$ \\
\hline $\mathrm{OR}(95 \% \mathrm{Cl})$ & $\begin{array}{l}1.00 \\
\text { (reference) }\end{array}$ & $\begin{array}{l}0.96(0.62- \\
1.48)\end{array}$ & $\begin{array}{l}0.69(0.36- \\
1.32)\end{array}$ & $\begin{array}{l}0.90(0.59- \\
1.35)\end{array}$ \\
\hline$P$-value & & 0.848 & 0.260 & 0.597 \\
\hline
\end{tabular}

BMI: body mass index, OR: odds ratio, Cl: confidence interval

*The $P$ Value $<0.05$

This study is aimed to investigate the association between MEG3 polymorphisms (rs3087918, rs11160608 rs7158663) and breast cancer. Our study recruited 1134 subjects containing 434 breast cancer patients and 700 healthy controls. The results indicated that the mutant homozygous GG of rs3087918 may associated with a decreased risk of $\mathrm{BC}$, especially in females age $<=49$. Comparison between case groups showed genotype GG and TG/GG of rs3087918 were correlated with her-2 receptor expression. The results of haplotype analysis for MEG3 showed that compared with wild haploid TAG, TCG haplotype may increase the risk of breast cancer, while other haplotypes were not significantly correlated with breast cancer risk. Furthermore, we found rs3087918 may influence the secondary structure of MEG3 and affect the bind of MEG3 to some miRNAs.
Table 4 Relationship between MEG3 rs3087918 and clinical characteristics of cases

\begin{tabular}{|c|c|c|c|c|}
\hline rs3087918 & $\pi$ & TG & GG & $\mathrm{TG}+\mathrm{GG}$ \\
\hline \multicolumn{5}{|l|}{ Age } \\
\hline$>49 /<=49$ & $102 / 69$ & $120 / 87$ & $28 / 19$ & $148 / 106$ \\
\hline $\mathrm{OR}(95 \% \mathrm{Cl})$ & $\begin{array}{l}1.00 \\
\text { (reference) }\end{array}$ & $\begin{array}{l}0.93(0.62- \\
1.408)\end{array}$ & $\begin{array}{l}1.00(0.52- \\
1.95)\end{array}$ & $\begin{array}{l}0.94(0.64- \\
1.40)\end{array}$ \\
\hline$P$-value & & 0.742 & 0.993 & 0.777 \\
\hline \multicolumn{5}{|l|}{ BMI $\left(k g / m^{2}\right)$} \\
\hline$>=24 /<24$ & $37 / 134$ & $60 / 147$ & $12 / 35$ & $72 / 182$ \\
\hline $\mathrm{OR}(95 \% \mathrm{Cl})$ & $\begin{array}{l}1.00 \\
\text { (reference) }\end{array}$ & $1.48(0.92-2.37)$ & $\begin{array}{l}1.24(0.59- \\
2.63)\end{array}$ & $\begin{array}{l}1.43(0.91- \\
2.26)\end{array}$ \\
\hline$P$-value & & 0.104 & 0.571 & 0.120 \\
\hline \multicolumn{5}{|c|}{ Menstrual status } \\
\hline yes/no & $114 / 57$ & $128 / 79$ & $29 / 18$ & $157 / 97$ \\
\hline $\mathrm{OR}(95 \% \mathrm{Cl})$ & $\begin{array}{l}1.00 \\
\text { (reference) }\end{array}$ & $0.81(0.53-1.24)$ & $\begin{array}{l}0.81(0.42- \\
1.59)\end{array}$ & $\begin{array}{l}0.81(0.54- \\
1.21)\end{array}$ \\
\hline$P$-value & & 0.330 & 0.526 & 0.307 \\
\hline \multicolumn{5}{|c|}{ Tumor size $(\mathrm{cm})$} \\
\hline$>2 /<=2$ & $85 / 86$ & $107 / 100$ & $31 / 16$ & $138 / 116$ \\
\hline OR( $(95 \% \mathrm{Cl})$ & $\begin{array}{l}1.00 \\
\text { (reference) }\end{array}$ & $1.08(0.72-1.62)$ & $\begin{array}{l}1.96(1.01- \\
3.92)\end{array}$ & $\begin{array}{l}1.20(0.82- \\
1.73)\end{array}$ \\
\hline$P$-value & & 0.701 & 0.050 & 0.350 \\
\hline \multicolumn{5}{|l|}{ Metastasis } \\
\hline $\begin{array}{c}\text { Positive/ } \\
\text { negtive }\end{array}$ & $93 / 78$ & $104 / 103$ & $24 / 23$ & $128 / 126$ \\
\hline OR( $(95 \% \mathrm{Cl})$ & $\begin{array}{l}1.00 \\
\text { (reference) }\end{array}$ & $0.85(0.56-1.27)$ & $\begin{array}{l}0.88(0.46- \\
1.68)\end{array}$ & $\begin{array}{l}0.85(0.58- \\
1.26)\end{array}$ \\
\hline$P$-value & & 0.422 & 0.686 & 0.419 \\
\hline \multicolumn{5}{|l|}{ Clinical Stage } \\
\hline |II-IV/I-II & $51 / 120$ & $59 / 148$ & $16 / 31$ & $75 / 179$ \\
\hline $\mathrm{OR}(95 \% \mathrm{Cl})$ & $\begin{array}{l}1.00 \\
\text { (reference) }\end{array}$ & $0.94(0.60-1.47)$ & $\begin{array}{l}1.21(0.60- \\
2.39)\end{array}$ & $\begin{array}{l}0.99(0.65- \\
1.51)\end{array}$ \\
\hline$P$-value & & 0.778 & 0.579 & 0.948 \\
\hline \multicolumn{5}{|l|}{ ER } \\
\hline $\begin{array}{c}\text { Positive/ } \\
\text { negtive }\end{array}$ & $115 / 56$ & $138 / 69$ & $33 / 14$ & $171 / 83$ \\
\hline OR(95\%Cl) & $\begin{array}{l}1.00 \\
\text { (reference) }\end{array}$ & $0.97(0.63-1.50)$ & $\begin{array}{l}1.15(0.58- \\
2.37)\end{array}$ & $\begin{array}{l}1.00(0.66- \\
1.51)\end{array}$ \\
\hline$P$-value & & 0.904 & 0.700 & 0.988 \\
\hline \multicolumn{5}{|l|}{ PR } \\
\hline $\begin{array}{c}\text { Positive/ } \\
\text { negtive }\end{array}$ & $94 / 77$ & $112 / 95$ & $33 / 14$ & $145 / 109$ \\
\hline $\mathrm{OR}(95 \% \mathrm{Cl})$ & $\begin{array}{l}1.00 \\
\text { (reference) }\end{array}$ & $0.97(0.64-1.45)$ & $\begin{array}{l}1.93(0.98- \\
3.97)\end{array}$ & $\begin{array}{l}1.09(0.74- \\
1.61)\end{array}$ \\
\hline$P$-value & & 0.867 & 0.063 & 0.666 \\
\hline \multicolumn{5}{|l|}{ Her-2 } \\
\hline $\begin{array}{c}\text { Positive/ } \\
\text { negtive }\end{array}$ & $62 / 109$ & $90 / 117$ & $27 / 20$ & $117 / 137$ \\
\hline $\mathrm{OR}(95 \% \mathrm{Cl})$ & $\begin{array}{l}1.00 \\
\text { (reference) }\end{array}$ & $1.35(0.89-2.05)$ & $\begin{array}{l}2.37(1.24- \\
4.63)\end{array}$ & $\begin{array}{l}1.50(1.01- \\
2.24)\end{array}$ \\
\hline$P$-value & & 0.155 & $0.01^{*}$ & $0.045^{*}$ \\
\hline
\end{tabular}

BMI: body mass index, ER: estrogen receptor, $P R$ : progesterone receptor, Her-2: human epidermal growth factor receptor-2, OR: odds ratio, $\mathrm{Cl}$ : confidence interval *The $P$ Value $<0.05$ 
Table 5 Haplotype analysis of MEG3 rs3087918

\begin{tabular}{lllll}
\hline Haplotypes & Control (\%) & Case (\%) & OR (95\%) & $P$ \\
\hline TAG & $293(41.89)$ & $155(37.44)$ & reference & - \\
GCG & $206(29.89)$ & $105(25.36)$ & $0.96(0.71-1.31)$ & 0.811 \\
TAA & $94(13.89)$ & $67(16.18)$ & $1.35(0.93-1.95)$ & 0.113 \\
GCA & $57(8.89)$ & $33(7.97)$ & $1.09(0.68-1.75)$ & 0.707 \\
TCG & $21(3.89)$ & $33(7.97)$ & $2.97(1.66-5.31)$ & $<0.001^{*}$ \\
\hline
\end{tabular}

The order of the three SNPs was rs3087918, rs11160608 rs 7158663. Haplotypes with frequency less than 0.03 were excluded. OR: odds ratio, Cl: confidence interval

*The $P$ Value $<0.05$

Previous evidences showed that MEG3 was highly expressed in normal tissues such as brain, pituitary, placenta and adrenal gland, and its transcripts can be detected in several human organs including ovary, testes, spleen, pancreas, liver, and mammary gland [7]. However, the expression of MEG3 was lower in various human tumors compared with that in normal human tissues, including breast cancer [24]. MEG3 was recognized as a tumor suppressor deponed on recent researches. In vitro experiments showed that restoring the expression of MEG3 could inhibit cancer cells proliferation and induce their apoptosis [25], and a similar tumor inhibition effect was found in nude mice [16]. MEG3 can also participate in epigenetic regulation of transcripts in the MEG3 region, such as DNA methylation [26, 27], snoRNA/microRNA regulation [2831]. It is also reported that SNPs in MEG3 gene have an influence on cancer risk. For example, Hou et al. observed a statistically significant increased risk between MEG3 rs11160608 and oral squamous cell carcinoma (OSCC) [24]. And Bayarmaa et al. found MEG3 polymorphisms were related to the chemotherapy response and toxicity of paclitaxel and cisplatin in breast cancer patients [32].
Moreover, Yang et al. found MEG3 rs7158663 have no association with lung cancer, while MEG3 rs4081134 was significantly influence the susceptibility of lung cancer in the Chinese population [33]. In this study, we found MEG3 rs3087918 was associated with a decreased breast cancer risk. We use a database named LncRNASNP2 (http:// bioinfo.life.hust.edu.cn/lncRNASNP/) to predict the potential function of rs3087918 on MEG3 gene. The results indicated that rs3087918 may influence MEG3 binding to miRNAs. In detail, rs3087918 may gain the targets of hsamiR-1203 to MEG3, while loss the target of hsa-miR-1393p and hsa-miR-5091 to MEG3. A study performed by Tomoyuki Okumura et al. found has-miR-1203 significantly associated with tumor recurrence [34]. Downregulation of has-miR-139-3p could induce cancer cell migration and invasion [35-37], and a pooled analysis proved that high hasmiR-139-3p expression was related to a better prognosis for hepatocellular carcinoma [38]. Thus, has-miR-139-3p was attributed as a tumor suppressor [39]. Hsa-miR-5091 was also reported as a biomarker with better prognosis for pancreatic ductal adenocarcinoma [40]. These were coincident with our results that rs3087918 was related to a decreased risk of breast cancer.

To be best of our knowledge, this is the first study to explore the association between MEG3 SNPs (rs3087918, rs11160608 rs7158663) and breast cancer risk. However, there are some potential limitations need to be clarified. First, we failed to consider the potential influence of environmental, lifestyle and other unknow risk factors on our study. Secondly, this is a one center case-control study with a small sample scale, we should not ignore the selective bias. In the future, more complete and larger sample scale study need to accomplish.

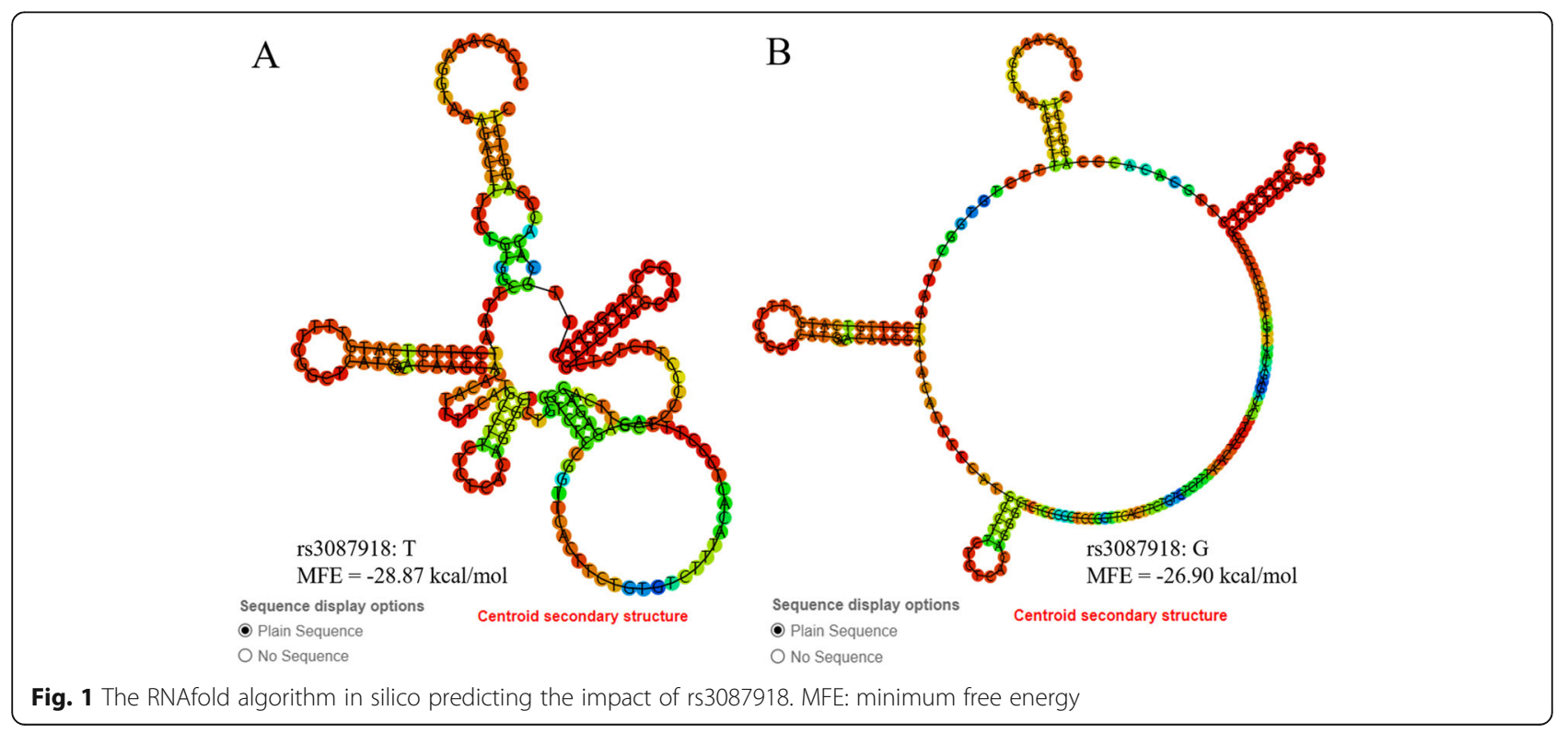




\section{Conclusion}

The wild-type homozygous GG of MEG3 rs3087918 was associated with a decreased risk of breast cancer. MEG3 haplotype TCG may increase the risk of breast cancer and it may owe to its effect on the structure and function of MEG3.

\section{Supplementary information}

Supplementary information accompanies this paper at https://doi.org/10 1186/s12885-020-07145-0.

Additional file 1: Figure S1. The prediction results of s3087918 affect the bind of MEG3 to miRNAs. (A) rs3087918 caused has-miR1203 target gain; (B) rs3087918 caused has-miR-139-3p target loss; (C) rs3087918 caused has-miR-5091 target loss. Table S1. Primers used for this study. Table S2. Stratified Analysis of rs11160608 and rs7158663 by age, BMI and menopausal status. Table $\mathbf{S 3}$. Association analysis between three SNPs inMEG3 and Molecular typing of breast cancer. Table S4. Rs3087918 influence MEG3 binding to miRNAs based on LnCRNASNP2 database.

\section{Abbreviations}

BC: Breast cancer; MEG3: Maternally expressed gene 3; IncRNA: long noncoding RNA; MAF: Minor allele frequency; HWE: Hardy-Weinberg Equilibrium; BMI: Body mass index; ORs: Odds ratios; Cls: 95\% confidence intervals

\section{Acknowledgements}

We thank all members of our study team for their whole-hearted cooperation and all included participants for their wonderful cooperation.

\section{Authors' contributions}

LK, ZYY, ZZ, and WY collected the samples. WSQ, XP, DYJ, LS, and LN detected the SNPS. DZJ and ZGX guided experiments. ZY and WM analyzed and interpreted the data. ZY was a major contributor in writing the manuscript. All authors read and approved the final manuscript.

\section{Funding}

Not applicable.

\section{Availability of data and materials}

The datasets generated during and/or analysed during the current study are available from the corresponding author on reasonable request.

\section{Ethics approval and consent to participate}

The protocol of this study was approved by the Ethics Committee of the Second Affiliated Hospital of Xi'an Jiaotong University Shaanxi Province (Xi'an, China). All patients gave written informed consent prior to participation in the study.

\section{Consent for publication}

Not applicable.

\section{Competing interests}

We declare no conflicts of interest for this study.

\section{Author details}

${ }^{1}$ Department of Obstetrics and Gynecology, The Second Affiliated Hospital of Xi'an Jiaotong University, Xi'an 710004, China. 'Department of Breast Surgery, The First Affiliated Hospital, College of Medicine, Zhejiang University, Hangzhou 310003, China. ${ }^{3}$ Department of Oncology, The Second Affiliated Hospital of Xi'an Jiaotong University, Xi'an 710004, China. ${ }^{4}$ Department of Hepatobiliary Surgery, The First Affiliated Hospital of Xi'an Jiaotong University, Xi'an 710061, China.
Received: 2 February 2020 Accepted: 7 July 2020

Published online: 15 July 2020

\section{References}

1. Bray F, Ferlay J, Soerjomataram I, Siegel RL, Torre LA, Jemal A. Global cancer statistics 2018: GLOBOCAN estimates of incidence and mortality worldwide for 36 cancers in 185 countries. CA Cancer J Clin. 2018;68(6):394-424.

2. Lehrer S, Green S, Rosenzweig KE. Affluence and breast Cancer. Breast J. 2016;22(5):564-7.

3. Veronesi U, Boyle P, Goldhirsch A, Orecchia R, Viale G. Breast cancer. Lancet. 2005:365(9472):1727-41

4. Gray JM, Rasanayagam S, Engel C, Rizzo J. State of the evidence 2017: an update on the connection between breast cancer and the environment. Environ Health. 2017;16(1):94.

5. Rodgers KM, Udesky JO, Rudel RA, Brody JG. Environmental chemicals and breast cancer: an updated review of epidemiological literature informed by biological mechanisms. Environ Res. 2018;160:152-82.

6. Miyoshi N, Wagatsuma H, Wakana S, Shiroishi T, Nomura M, Aisaka K, Kohda T, Surani MA, Kaneko-Ishino T, Ishino F. Identification of an imprinted gene, Meg3/Gtl2 and its human homologue MEG3, first mapped on mouse distal chromosome 12 and human chromosome 14q. Genes Cells. 2000;5(3):21120.

7. Zhou Y, Zhang X, Klibanski A. MEG3 noncoding RNA: a tumor suppressor. J Mol Endocrinol. 2012:48(3):R45-53.

8. da Rocha ST, Edwards CA, Ito M, Ogata T, Ferguson-Smith AC. Genomic imprinting at the mammalian Dlk1-Dio3 domain. Trends Genet. 2008;24(6): 306-16.

9. Zhang X, Rice K, Wang Y, Chen W, Zhong Y, Nakayama Y, Zhou Y, Klibanski A. Maternally expressed gene 3 (MEG3) noncoding ribonucleic acid: isoform structure, expression, and functions. Endocrinology. 2010;151(3):939-47.

10. Wei GH, Wang X. IncRNA MEG3 inhibit proliferation and metastasis of gastric cancer via p53 signaling pathway. Eur Rev Med Pharmacol Sci. 2017; 21(17):3850-6.

11. Zhang J, Yao T, Wang Y, Yu J, Liu Y, Lin Z. Long noncoding RNA MEG3 is downregulated in cervical cancer and affects cell proliferation and apoptosis by regulating miR-21. Cancer Biol Ther. 2016;17(1):104-13.

12. Sun $L$, Li Y, Yang B. Downregulated long non-coding RNA MEG3 in breast cancer regulates proliferation, migration and invasion by depending on p53's transcriptional activity. Biochem Biophys Res Commun. 2016:478(1): 323-9.

13. He Y, Luo Y, Liang B, Ye L, Lu G, He W. Potential applications of MEG3 in cancer diagnosis and prognosis. Oncotarget. 2017;8(42):73282-95.

14. Li J, Zi Y, Wang W, Li Y. Long noncoding RNA MEG3 inhibits cell proliferation and metastasis in chronic myeloid leukemia via targeting miR184. Oncol Res. 2018;26(2):297-305

15. Zhang W, Shi S, Jiang J, Li X, Lu H, Ren F. LncRNA MEG3 inhibits cell epithelial-mesenchymal transition by sponging miR-421 targeting Ecadherin in breast cancer. Biomed Pharmacother. 2017:91:312-9.

16. Zhuo H, Tang J, Lin Z, Jiang R, Zhang X, Ji J, Wang P, Sun B. The aberrant expression of MEG3 regulated by UHRF1 predicts the prognosis of hepatocellular carcinoma. Mol Carcinog. 2016;55(2):209-19.

17. Auton A, Brooks LD, Durbin RM, Garrison EP, Kang HM, Korbel JO, Marchini JL, McCarthy S, McVean GA, Abecasis GR. A global reference for human genetic variation. Nature. 2015;526(7571):68-74.

18. Michailidou K, Beesley J, Lindstrom S, Canisius S, Dennis J, Lush MJ, Maranian MJ, Bolla MK, Wang Q, Shah M, et al. Genome-wide association analysis of more than 120,000 individuals identifies 15 new susceptibility loci for breast cancer. Nat Genet. 2015;47(4):373-80.

19. Pellegrino B, Bella M, Michiara M, Zanelli P, Naldi N, Porzio R, Bortesi B, Boggiani D, Zanoni D, Camisa R, et al. Triple negative status and BRCA mutations in contralateral breast cancer: a population-based study. Acta Biomed. 2016;87(1):54-63.

20. Goovaerts T, Steyaert S, Vandenbussche CA, Galle J, Thas O, Van Criekinge W, De Meyer T. A comprehensive overview of genomic imprinting in breast and its deregulation in cancer. Nat Commun. 2018:9(1):4120.

21. Cao X, Zhuang S, Hu Y, Xi L, Deng L, Sheng H, Shen W. Associations between polymorphisms of long non-coding RNA MEG3 and risk of colorectal cancer in Chinese. Oncotarget. 2016:7(14):19054-9.

22. Zhuo ZJ, Zhang R, Zhang J, Zhu J, Yang T, Zou Y, He J, Xia H. Associations between IncRNA MEG3 polymorphisms and neuroblastoma risk in Chinese children. Aging. 2018;10(3):481-91. 
23. Miao YR, Liu W, Zhang Q, Guo AY. IncRNASNP2: an updated database of functional SNPs and mutations in human and mouse IncRNAs. Nucleic Acids Res. 2018:46(D1):D276-80.

24. Hou Y, Zhang B, Miao L, Ji Y, Yu Y, Zhu L, Ma H, Yuan H. Association of long non-coding RNA MEG3 polymorphisms with oral squamous cell carcinoma risk. Oral Dis. 2019;25(5):1318-24.

25. Xiu YL, Sun KX, Chen X, Chen S, Zhao Y, Guo QG, Zong ZH. Upregulation of the IncRNA Meg3 induces autophagy to inhibit tumorigenesis and progression of epithelial ovarian carcinoma by regulating activity of ATG3. Oncotarget. 2017;8(19):31714-25.

26. Zhao J, Dahle D, Zhou Y, Zhang X, Klibanski A. Hypermethylation of the promoter region is associated with the loss of MEG3 gene expression in human pituitary tumors. J Clin Endocrinol Metab. 2005;90(4):2179-86.

27. Benetatos L, Dasoula A, Hatzimichael E, Georgiou I, Syrrou M, Bourantas KL. Promoter hypermethylation of the MEG3 (DLK1/MEG3) imprinted gene in multiple myeloma. Clin Lymphoma Myeloma. 2008;8(3):171-5.

28. Ueda T, Volinia S, Okumura H, Shimizu M, Taccioli C, Rossi S, Alder H, Liu CG, Oue N, Yasui W, et al. Relation between microRNA expression and progression and prognosis of gastric cancer: a microRNA expression analysis. Lancet Oncol. 2010;11(2):136-46.

29. Shih KK, Qin LX, Tanner EJ, Zhou Q, Bisogna M, Dao F, Olvera N, Viale A, Barakat RR, Levine DA. A microRNA survival signature (MiSS) for advanced ovarian cancer. Gynecol Oncol. 2011;121(3):444-50.

30. Sahoo T, del Gaudio D, German JR, Shinawi M, Peters SU, Person RE, Garnica A, Cheung SW, Beaudet AL. Prader-Willi phenotype caused by paternal deficiency for the HBII-85 C/D box small nucleolar RNA cluster. Nat Genet. 2008;40(6):719-21.

31. Bortolin-Cavaille ML, Cavaille J. The SNORD115 (H/MBII-52) and SNORD116 ( $\mathrm{H} / \mathrm{MBII}-85$ ) gene clusters at the imprinted Prader-Willi locus generate canonical box C/D snoRNAs. Nucleic Acids Res. 2012;40(14):6800-7.

32. Bayarmaa B, Wu Z, Peng J, Wang Y, Xu S, Yan T, Yin W, Lu J, Zhou L. Association of LncRNA MEG3 polymorphisms with efficacy of neoadjuvant chemotherapy in breast cancer. BMC Cancer. 2019;19(1):877.

33. Yang Z, Li H, Li J, Lv X, Gao M, Bi Y, Zhang Z, Wang S, Li S, Li N, et al. Association between long noncoding RNA MEG3 polymorphisms and lung Cancer susceptibility in Chinese northeast population. DNA Cell Biol. 2018; 37(10):812-20

34. Okumura T, Shimada Y, Omura T, Hirano K, Nagata T, Tsukada K. MicroRNA profiles to predict postoperative prognosis in patients with small cell carcinoma of the esophagus. Anticancer Res. 2015;35(2):719-27.

35. Yonemori M, Seki N, Yoshino H, Matsushita R, Miyamoto K, Nakagawa M, Enokida H. Dual tumor-suppressors miR-139-5p and miR-139-3p targeting matrix metalloprotease 11 in bladder cancer. Cancer Sci. 2016;107(9):1233-42.

36. Ng L, Wan TM, Man JH, Chow AK, lyer D, Chen G, Yau TC, Lo OS, Foo DC, Poon JT, et al. Identification of serum miR-139-3p as a non-invasive biomarker for colorectal cancer. Oncotarget. 2017;8(16):27393-400.

37. Sannigrahi MK, Sharma R, Singh V, Panda NK, Rattan V, Khullar M. Role of host miRNA Hsa-miR-139-3p in HPV-16-induced carcinomas. Clin Cancer Res. 2017;23(14):3884-95.

38. Zhu Y, Zhou C, He Q. High miR-139-3p expression predicts a better prognosis for hepatocellular carcinoma: a pooled analysis. J Int Med Res. 2019:47(1):383-90.

39. Zhang W, Xu J, Wang K, Tang X, He J. miR-139-3p suppresses the invasion and migration properties of breast cancer cells by targeting RAB1A. Oncol Rep. 2019;42(5):1699-708.

40. Liao X, Wang X, Huang K, Yang C, Yu T, Han C, Zhu G, Su H, Huang R, Peng T. Genome-scale analysis to identify prognostic microRNA biomarkers in patients with early stage pancreatic ductal adenocarcinoma after pancreaticoduodenectomy. Cancer Manag Res. 2018;10:2537-51.

\section{Publisher's Note}

Springer Nature remains neutral with regard to jurisdictional claims in published maps and institutional affiliations.

Ready to submit your research? Choose BMC and benefit from:

- fast, convenient online submission

- thorough peer review by experienced researchers in your field

- rapid publication on acceptance

- support for research data, including large and complex data types

- gold Open Access which fosters wider collaboration and increased citations

- maximum visibility for your research: over $100 \mathrm{M}$ website views per year

At $\mathrm{BMC}$, research is always in progress.

Learn more biomedcentral.com/submissions 